\title{
Null-solutions for partial differential operators with several Fuchsian variables
}

\author{
By \\ Malika BELARBI, Takeshi MANDAI, and Mustapha MECHAB
}

\begin{abstract}
K. Igari has constructed a distribution null-solution for Fuchsian partial differential operators in the sense of Baouendi-Goulaouic, that is, operators with one Fuchsian variable. Our aim is to extend his result for operators having several Fuchsian variables with analytic coefficients defined by N. S. Madi.
\end{abstract}

\section{Introduction}

Let $P$ be a linear differential operator in $\mathbb{C}^{n}$ and set $S=\left\{x \in \mathbb{C}^{n} ; \phi(x)=0\right\}$, with $\operatorname{grad} \phi \neq 0$, a hypersurface in $\mathbb{C}^{n}$. Since the work of Cauchy ([2]-[4]), the Cauchy problem has been abundantly studied. One of the questions related to this problem was the uniqueness of the solution. The answer to this question depends on the nature of the hypersurface of data. In the holomorphic noncharacteristic case, the uniqueness of the holomorphic solution results from the Cauchy-Kowalevsky theorem. For the characteristc case, Petrowsky [15], in 1939, gave examples of operators admitting several regular solutions. In the linear case,

2000 Mathematics Subject Classification. Primary 35D05; Secondary 35A07, 35A20.

Key words and phrases. several Fuchsian variables, distribution null-solutions.

The research was partially supported by MESR within the framework of the research projects B*2201/04/02 and CMEP 02 MDU 545. The research was also partially supported by the Ministry of Education, Culture, Sports, Science and Technology (Japan), Grant-in-Aid for Scientific Research (C), 12640194, 2000-2002. 
the proof of nonuniqueness of the solution consist in finding a solution of the differential equation which is null on only one side of the hypersurface of the data. This type of solutions are usually called null-solutions of the differential equation ([14]). When the hypersurface $S$ of the data is considered to be $\{t=0\}$ in $\mathbb{C}_{(t, x)}^{n+1}$, a typical example of characteristic Cauchy problems is given by that to Fuchsian partial differential equations, which is a natural extension of ordinary differential equations with regular singularity at a point, and are also called differential equations with regular singularity along the initial hypersurface. Fuchsian partial differential operators were defined by M. S. Baouendi and C. Goulaouic [1], and they established fundamental theorems on the characteristic Cauchy problems for Fuchsian equations: Cauchy-Kowalevsky type theorem and Holmgren type theorem, which are natural extensions of the classical theorems for noncharacteristic Cauchy problems. One can also quote some works of H. Tahara, who extensively studied the characteristic problems, in particular that related to Fuchsian operators ([16]-[21]). Fuchsian operators with holomorphic coefficients do not have sufficiently smooth null-solutions, while K. Igari [5] showed the existence of distribution null-solutions $u(t, x)$ under an additional condition. His null-solutions are analytic in $x$. In [11], the second author removed the condition of K. Igari.

At the beginning of the eighties, N. S. Madi generalized the concept of Fuchsian operator to the case of several Fuchsian variables ([8], [9], [10]). In many situations, he established the existence and the uniqueness of the solution of the Goursat problem, which assigns data on several hypersurfaces, typically $\{x=0\}$ in $\mathbb{C}_{(x, y)}^{p+q}$, in spaces of holomorphic or smooth functions.

In this work, we extend the result of K. Igari to a class of operators with several Fuchsian variables. The method to construct distribution solutions on $\mathbb{R}_{(x, y)}^{p+q}$ which is analytic in $y$ is similar to that used in [12] and [13]. We construct distribution solutions in the form $u(x, y)=\int_{\Gamma} \mathcal{G}(\zeta ; x) V(x, y ; \zeta) d \zeta$, where $\Gamma$ is a suitably chosen closed curve in $\mathbb{C}^{p}, \mathcal{G}(\zeta ; x)$ is a basic distribution of $x \in \mathbb{R}^{p}$ with holomorphic parameters $\zeta \in \mathbb{C}^{p}$, and $V$ is a holomorphic function of $(x, y ; \zeta)$ in a neighborhood of $\{(0,0)\} \times \Gamma$. 
In the case of several Fuchsian variables, there may exist a $C^{\infty}$ null-solutions, contrary to the case of one Fuchsian variable. We give some simple examples in the last section.

\section{Main result}

First, we fix some notation.

\section{Notation:}

- $\mathbb{N}$ denotes the set of nonnegative integers. Set $\mathbb{N}^{*}:=\mathbb{N} \backslash\{0\}$.

- For a multi-index $\alpha=\left(\alpha_{1}, \ldots, \alpha_{p}\right) \in \mathbb{N}^{p}$, we set $|\alpha|:=\alpha_{1}+\cdots+\alpha_{p}$. For $x=\left(x_{1}, \ldots, x_{p}\right) \in \mathbb{C}^{p}$, we write $x^{\alpha}:=x_{1}^{\alpha_{1}} \ldots x_{p}^{\alpha_{1}}$. Similarly, $D_{x_{i}}:=\partial / \partial x_{i}$, $D_{x}^{\alpha}:=D_{x_{1}}^{\alpha_{1}} \ldots D_{x_{p}}^{\alpha_{p}}$, and so on, as usual.

For a nonempty set $\Omega \subset \mathbb{C}^{q}$, the set of holomorphic functions on (a neighborhood of) $\Omega$ is denoted by $\mathscr{O}(\Omega)$.

- For multi-indices $\alpha, \beta \in \mathbb{N}^{p}$, we write $\alpha \leq \beta$ if $\alpha_{i} \leq \beta_{i}$ for every $i$, and $\alpha<\beta$ if $\alpha \leq \beta$ and $\alpha \neq \beta$. We say $\alpha$ is smaller than $\beta$, if $\alpha<\beta$.

- We write $x \cdot y:=\sum_{i} x_{i} \overline{y_{i}}$ and $|x|:=\sqrt{\sum_{i}\left|x_{i}\right|^{2}}$. An open ball is denoted by $B_{r}(a):=\left\{x \in \mathbb{C}^{p}|| x-a \mid<r\right\}$ for $a \in \mathbb{C}^{p}$ and $r>0$. We use the same notation for any dimension. The closure of a set $A$ is denoted by $\bar{A}$.

- For an open set $\mathcal{V} \subset \mathbb{R}^{p}$, the set of $C^{\infty}$ functions on $\mathcal{V}$ with compact support is denoted by $\mathscr{D}(\mathcal{V})=C_{0}^{\infty}(\mathcal{V})$. We set

$$
\mathscr{D}^{\prime}(\mathcal{V} ; \mathscr{O}(\Omega)):=\mathscr{L}(\mathscr{D}(\mathcal{V}), \mathscr{O}(\Omega))
$$

$(\mathscr{O}(\Omega)$-valued distributions), where $\mathscr{L}(E, F)$ denotes the set of continuous linear mappings from $E$ to $F$. The value of $f \in \mathscr{D}^{\prime}(\mathcal{V} ; \mathscr{O}(\Omega))$ at $g \in \mathscr{D}(\mathcal{V})$ is denoted by $\langle f, g\rangle=\langle f(x, y), g(x)\rangle_{x}$, which is also used as the integral $\int_{\mathcal{V}} f(x, y) g(x) d x$ for usual functions $f$ and $g$. Similarly, we use the space 
$\mathscr{S}\left(\mathbb{R}^{p}\right)$ of rapidly decreasing $C^{\infty}$ functions and the space $\mathscr{S}^{\prime}\left(\mathbb{R}^{p} ; \mathscr{O}(\Omega)\right):=$ $\mathscr{L}\left(\mathscr{S}\left(\mathbb{R}^{p}\right), \mathscr{O}(\Omega)\right)$ of $\mathscr{O}(\Omega)$-valued tempered distributions.

We write $\mathbb{R}_{+}:=(0, \infty), \mathbb{R}_{+}^{p}:=\left(\mathbb{R}_{+}\right)^{p}$, and we set $\mathcal{V}_{+}:=\mathcal{V} \cap \mathbb{R}_{+}^{p}$. We also set

$$
\mathscr{D}_{+}^{\prime}(\mathcal{V} ; \mathscr{O}(\Omega)):=\left\{f \in \mathscr{D}^{\prime}(\mathcal{V} ; \mathscr{O}(\Omega)) \mid \operatorname{supp}_{x} f \subset \mathcal{V} \cap \overline{\mathbb{R}_{+}^{p}}\right\}
$$

where $\operatorname{supp}_{x} f$ is the support of $f$ with respect to $x \in \mathcal{V}$. We also use $C_{+}^{N}(\mathcal{V} ; \mathscr{O}(\Omega)), \mathscr{S}_{+}^{\prime}\left(\mathbb{R}^{p} ; \mathscr{O}(\Omega)\right)$ and so on, similarly.

Now, consider the following differential operator

$$
\mathcal{P}=\sum_{|\alpha|+|\beta| \leq m} a_{\alpha, \beta}(x, y) D_{x}^{\alpha} D_{y}^{\beta}
$$

in $(x, y) \in \mathbb{C}^{p} \times \mathbb{C}^{q}$, where $a_{\alpha, \beta}$ are holomorphic functions in a neighborhood of the origin $(0,0)$.

Definition 2.1. ([10]) (1) The weight of a monomial $a_{\alpha, \beta} D_{x}^{\alpha} D_{y}^{\beta}$ with respect to $x$ is the smallest $\tau \in \mathbb{Z}^{p}$ such that $a_{\alpha, \beta}=O\left(x^{\alpha-\tau}\right)$ and $\alpha-\tau \in \mathbb{N}^{p}$, where $f=O\left(x^{\gamma}\right)$ means that $f(x, y) / x^{\gamma}$ is bounded as $x \rightarrow 0$, or equivalently $f(x, y)=x^{\gamma} \widetilde{f}(x, y)$ for some holomorphic function $\widetilde{f}$.

(2) We say that $\mathcal{P}$ is a Fuchsian operator with weight $\mu \in \mathbb{N}^{p}$ with respect to $x$, if the following three conditions are satisfied.

$\left(\mathrm{H}_{1}\right)$ The weight of every monomial $a_{\alpha, \beta}(x, y) D_{x}^{\alpha} D_{y}^{\beta}$ is less than or equal to $\mu$. In other words, there exists a holomorphic function $\widetilde{a}_{\alpha, \beta}$ such that $a_{\alpha, \beta}(x, y)=$ $x^{\alpha-\mu} \widetilde{a}_{\alpha, \beta}(x, y)$.

$\left(\mathrm{H}_{2}\right)$ If $\beta \neq 0$, then the weight of monomial $a_{\alpha, \beta}(x, y) D_{x}^{\alpha} D_{y}^{\beta}$ is smaller than $\mu$. In other words, if $\beta \neq 0$, then $\widetilde{a}_{\alpha, \beta}(x, y)=O\left(x^{\gamma}\right)$ for some $\gamma>0$.

$\left(\mathrm{H}_{3}\right)$ If $\mu_{i} \neq 0$ and $\beta \neq 0$, then the weight with respect to $x_{i}$ of each monomial $a_{\alpha, \beta} D_{x}^{\alpha} D_{y}^{\beta}$ is smaller than $\mu_{i}$.

It might be better to add another condition that $\mathcal{P}$ has at least one monomial $x^{\alpha-\mu} \widetilde{a}_{\alpha, 0}(x, y) D_{x}^{\alpha}$ such that $|\alpha|=m$ and $\widetilde{a}_{\alpha, 0}(0,0) \neq 0$. In the case of one Fuchsian 
variable, this condition is included in the definition of Fuchsian operator in [1]. This condition, however, is implied by the condition $\left(\mathrm{A}_{0}\right)$ below. Hence, we adopt Madi's definition.

Though we assume that the weight of $\mathcal{P}$ is 0 later, we are giving a general setting here.

Example 2.1. Set $x D_{x}:=\left(x_{1} D_{x_{1}}, \ldots, x_{p} D_{x_{p}}\right)$. The operator (2.1) is a Fuchsian operator with weight 0 with respect to $x$ if and only if $\mathcal{P}$ can be written as

$$
\mathcal{P}=\sum_{|\alpha|+|\beta| \leq m} b_{\alpha, \beta}(x, y)\left(x D_{x}\right)^{\alpha} D_{y}^{\beta},
$$

$b_{\alpha, \beta}$ are holomorphic, and if $\beta \neq 0$ then $b_{\alpha, \beta}(x, y)=O\left(x^{\gamma}\right)$ for some $\gamma>0$.

Definition 2.2. ([10]) (1) For $w \in \mathbb{C}$ and $l \in \mathbb{N}$, we define $\mathcal{C}_{l}(w)$ by $\mathcal{C}_{0}(w):=1$ and $\mathcal{C}_{l}(w):=\prod_{j=0}^{l-1}(w-j)$. For $\lambda \in \mathbb{C}^{p}$ and $\alpha \in \mathbb{N}^{p}$, we set $\mathcal{C}_{\alpha}(\lambda):=\prod_{i=1}^{p} \mathcal{C}_{\alpha_{i}}\left(\lambda_{i}\right)$. (2) Let $\mathcal{P}$ be a Fuchsian operator. By the condition $\left(\mathrm{H}_{1}\right)$, there exist holomorphic functions $\widetilde{a}_{\alpha, 0}$ such that $a_{\alpha, 0}(x, y)=x^{\alpha-\mu} \widetilde{a}_{\alpha, 0}(x, y)$. Then, the Fuchsian characteristic polynomial of $\mathcal{P}$ is defined by

$$
\widetilde{Q}(y ; \lambda):=\sum_{\alpha \geq \mu} \widetilde{a}_{\alpha, 0}(0, y) \mathcal{C}_{\alpha-\mu}(\lambda)
$$

Note that $\left.\left(x^{-\lambda} \mathcal{P} x^{\lambda+\mu}\right)\right|_{x=0}=\widetilde{Q}(y ; \lambda) \mathcal{C}_{\mu}(\lambda+\mu)$.

Definition 2.3. We say that a Fuchsian operator $\mathcal{P}$ satisfies the condition $\left(\mathrm{A}_{0}\right)$, if there exist a domain $\Omega$ including $y=0$ in $\mathbb{C}^{q}$, a holomorphic function $\lambda_{0} \in \mathscr{O}(\Omega)$, and a constant $c>0$ such that

$$
\begin{aligned}
& \widetilde{Q}\left(y ; \lambda_{0}(y)\right) \equiv 0 \quad \text { on } \Omega \\
& \left|\widetilde{Q}\left(0 ; \lambda_{0}(0)+k\right)\right| \geq c(1+|k|)^{m-|\mu|} \quad \text { for every } k \in\left(\mathbb{N}^{p}\right)^{*}
\end{aligned}
$$

where $\left(\mathbb{N}^{p}\right)^{*}:=\mathbb{N}^{p} \backslash\{0\}$.

If $p=1$, then the condition (2.4) reduces to

$$
\begin{gathered}
\widetilde{a}_{m, 0}(0,0) \neq 0 \\
\widetilde{Q}\left(0 ; \lambda_{0}(0)+k\right) \neq 0 \quad \text { for every } k \in \mathbb{N}^{*}
\end{gathered}
$$

and hence the condition $\left(\mathrm{A}_{0}\right)$ is the condition of Igari in [5]. 
Example 2.2. Let $f_{i}\left(y ; \lambda_{i}\right)$ be a polynomial of $\lambda_{i}$ of degree $m-|\mu|$ having the following three properties.

(a) The coefficients are holomorphic functions of $y$.

(b) At $y=0$, the coefficients are all real and the coefficient of $\lambda_{i}^{m-|\mu|}$ is positive.

(c) At $y=0$, the polynomial $f_{i}\left(0, \lambda_{i}\right)$ has at least one real zero and the largest real zero is simple.

If $\widetilde{Q}(y ; \lambda)=\sum_{i=1}^{p} f_{i}\left(y ; \lambda_{i}\right)$ with $f_{i}$ having the above three properties, then the condition $\left(\mathrm{A}_{0}\right)$ is satisfied.

In fact, we can take a holomorphic $\lambda_{0, i}(y)$ in a neighborhood of $y=0$ such that $\lambda_{0, i}(0)$ is the largest real zero in $(\mathrm{c})$ and $f_{i}\left(y ; \lambda_{0, i}(y)\right) \equiv 0$. By (b), there exists a positive $c$ such that $f_{i}\left(0 ; \lambda_{0, i}(0)+k_{i}\right) \geq c\left(1+k_{i}\right)^{m-|\mu|}$ for every $k_{i} \in \mathbb{N}^{*}$. Since there exists a constant $c^{\prime}>0$ such that $\left(1+k_{1}\right)^{m-|\mu|}+\cdots+\left(1+k_{r}\right)^{m-|\mu|} \geq$ $c^{\prime}(1+|k|)^{m-|\mu|}$ for every $k \in \mathbb{N}^{r}$ and $1 \leq r \leq p$, we have the inequality (2.4) for $\widetilde{Q}(0 ; \lambda)=\sum_{i=1}^{p} f_{i}\left(0 ; \lambda_{i}\right)$.

Definition 2.4. A distribution $u$ in a neighborhood of $(0,0) \in \mathbb{R}^{p} \times \mathbb{R}^{q}$ is called a null-solution of $\mathcal{P}$ at $(0,0)$ with respect to $\overline{\mathbb{R}_{+}^{p}} \times \mathbb{R}^{q}$, if $\mathcal{P} u=0$ in a neighborhood of $(0,0)$ and

$$
(0,0) \in \operatorname{supp} u \subset \overline{\mathbb{R}_{+}^{p}} \times \mathbb{R}^{q}
$$

where $\operatorname{supp} u$ is the support of $u$.

Now, we can give our main theorem.

Theorem 2.1. $\quad$ Assume that $\mathcal{P}$ is a Fuchsian operator with weight 0 with respect to $x$ and that $\mathcal{P}$ satisfies the condition $\left(\mathrm{A}_{0}\right)$. Then, there exist an open neighborhood $\mathcal{V}$ of 0 in $\mathbb{R}^{p}$, a domain $\Omega$ including 0 in $\mathbb{C}^{q}$, and a null-solution $u \in \mathscr{D}_{+}^{\prime}(\mathcal{V} ; \mathscr{O}(\Omega)) \cap$ $C^{\infty}\left(\mathcal{V}_{+} ; \mathscr{O}(\Omega)\right)$ of $\mathcal{P}$ at $(0,0)$ with respect to $\overline{\mathbb{R}_{+}^{p}} \times \mathbb{R}^{q}$.

From now on, we assume $p \geq 2$, since we have a far better result ([12]) when $p=1$. 


\section{Preliminaries}

In this section, we give some preliminary results needed for the later argument.

Definition 3.1. For $w \in \mathbb{C}$ with $\operatorname{Re} w>-1$ and $t \in \mathbb{R}$, we set

$$
G(w ; t):=\frac{t_{+}^{w}}{\Gamma(w+1)}, \quad t_{+}:=\left\{\begin{array}{ll}
t & (t>0) \\
0 & (t \leq 0)
\end{array},\right.
$$

where $\Gamma(w)$ is the Gamma function. Since $G(w ; \cdot)$ is a locally integrable function of polynomial growth, it is a tempered distribution, with a holomorphic parameter $w$. Since $G(w ; t)=D_{t} G(w+1 ; t)$, we can extend $G$ to the whole $w \in \mathbb{C}$ holomorphically. Thus, we have

$$
G(w ; t) \in \mathscr{S}_{+}^{\prime}(\mathbb{R} ; \mathscr{O}(\mathbb{C})) \bigcap C^{\infty}\left(\mathbb{R}_{+} ; \mathscr{O}(\mathbb{C})\right)
$$

Note that $G(-d ; t)=\delta^{(d-1)}(t)$ if $d \in \mathbb{N}^{*}$.

The distribution $G(w ; t)$ has the following basic properties. We omit the proof of this lemma, since it is very easy.

Lemma 3.1. (1) For $l \in \mathbb{N}$, we have $D_{t}^{l} G(w ; t)=G(w-l ; t)$ and $t^{l} G(w ; t)=$ $\mathcal{C}_{l}(w+l) G(w+l ; t)$.

(2) For a polynomial $E(\lambda)$, we have $E\left(t D_{t}\right) G(w ; t)=E(w) G(w ; t)$.

(3) For $\epsilon>0$, we have $\langle G(w ; t), \exp (-t / \epsilon)\rangle_{t}=\epsilon^{w+1}$. Note that

$$
\langle G(w ; t), \exp (-t / \epsilon)\rangle_{t}=\langle G(w ; t), \phi(t) \exp (-t / \epsilon)\rangle_{t}
$$

is well-defined, where $\phi \in C^{\infty}(\mathbb{R}), \phi(t)=1$ in a neighborhood of $[0, \infty)$, and $\inf \operatorname{supp} \phi>-\infty$, although $\exp (-t / \epsilon) \notin \mathscr{S}(\mathbb{R})$.

For a higher dimension $(p>1)$, we define

$$
\mathcal{G}(z ; x):=\bigotimes_{i=1}^{p} G\left(z_{i} ; x_{i}\right) \in \mathscr{S}_{+}^{\prime}\left(\mathbb{R}^{p} ; \mathscr{O}\left(\mathbb{C}^{p}\right)\right) \cap C^{\infty}\left(\mathbb{R}_{+}^{p} ; \mathscr{O}\left(\mathbb{C}^{p}\right)\right)
$$

From Lemma 3.1, we easily get the following. 
Lemma 3.2. (1) For $\alpha \in \mathbb{N}^{p}$, we have $D_{x}^{\alpha} \mathcal{G}(z ; x)=\mathcal{G}(z-\alpha ; x)$ and $x^{\alpha} \mathcal{G}(z ; x)=$ $\mathcal{C}_{\alpha}(z+\alpha) \mathcal{G}(z+\alpha ; x)$.

(2) For a polynomial $F(\lambda)$, we have $F\left(x D_{x}\right) \mathcal{G}(z ; x)=F(z) \mathcal{G}(z ; x)$.

(3) For $\eta \in \mathbb{R}_{+}^{p}$, we have $\left\langle\mathcal{G}(z ; x), \exp \left(-\sum_{i=1}^{p} x_{i} / \eta_{i}\right)\right\rangle_{x}=\eta^{z+\mathbb{I}}$, where $\mathbb{I}:=$ $(1, \ldots, 1) \in \mathbb{N}^{p}$.

We close this section by giving Madi's result, which will be used in the proof of our theorem.

Theorem 3.1. ([10]) Let $\mathcal{P}$ be a Fuchsian operator with weight $\mu$ with respect to $x$. If there exist a positive constant $c$ and a neighborhood $\Omega$ of $y=0$ such that

$$
|\widetilde{Q}(y ; k)| \geq c(1+|k|)^{m-|\mu|} \quad \text { for every } k \in \mathbb{N}^{p} \text { and } y \in \Omega
$$

then the local Goursat problem

$$
\left\{\begin{array}{l}
\mathcal{P} u(x, y)=f(x, y) \\
u(x, y)-w(x, y)=O\left(x^{\mu}\right)
\end{array}\right.
$$

has a unique holomorphic solution $u(x, y)$ in a neighborhood of $(0,0)$ for every given data $f(x, y)$ and $w(x, y)$ holomorphic in a neighborhood of $(0,0)$.

Note that if the weight $\mu=0$, then the equation $\mathcal{P} u=f$ determines the holomorphic solution $u$ uniquely without giving $w$.

\section{Construction of a solution}

In this section, we assume the assumptions of Theorem 2.1, and construct a solution. The idea to construct our solution is very simple: we construct a solution in the form

$$
u(x, y)=\int_{\Gamma} \mathcal{G}(\zeta ; x) V(x, y ; \zeta) d \zeta
$$

where $\Gamma:=\Gamma_{1} \times \cdots \times \Gamma_{p}$, each $\Gamma_{i}$ is a suitable simple closed curve in $\mathbb{C}, d \zeta:=$ $d \zeta_{1} \ldots d \zeta_{p}$, and $V$ is holomorphic in a neighborhood of $\{(0,0)\} \times \Gamma$. This is a simple extension of the idea used in [13]. 
Since we are assuming that the weight of $\mathcal{P}$ is 0 , we can write $\mathcal{P}$ as

$$
\mathcal{P}=p\left(x, y ; x D_{x}, D_{y}\right)=\sum_{|\alpha|+|\beta| \leq m} b_{\alpha, \beta}(x, y)\left(x D_{x}\right)^{\alpha} D_{y}^{\beta},
$$

where $b_{\alpha, \beta}$ are holomorphic in a neighborhood of $(0,0)$. Since

$$
x D_{x}\{\mathcal{G}(\zeta ; x) V(x, y ; \zeta)\}=\mathcal{G}(\zeta ; x)\left(x D_{x}+\zeta\right) V(x, y ; \zeta)
$$

by Lemma $3.2(2)$, we have

$$
\mathcal{P} u(x, y)=\int_{\Gamma} \mathcal{G}(\zeta ; x)\left\{p\left(x, y ; x D_{x}+\zeta, D_{y}\right) V(x, y ; \zeta)\right\} d \zeta .
$$

We want to take $V$ such that

$$
p\left(x, y ; x D_{x}+\zeta, D_{y}\right) V(x, y ; \zeta)=\frac{\widetilde{Q}(y ; \zeta)}{\left(\zeta_{1}-\lambda_{0,1}(y)\right) \cdots\left(\zeta_{p}-\lambda_{0, p}(y)\right)}
$$

in a neighborhood of $\{(0,0)\} \times \Gamma$.

First, we prepare two lemmas. We set $\stackrel{\AA}{\lambda}:=\lambda_{0}(0)$.

Lemma 4.1. There exists $\epsilon>0$ such that if $\lambda \in B_{\epsilon}(\stackrel{\circ}{\lambda})$ and $y \in B_{\epsilon}(0)$ then

$$
|\widetilde{Q}(y ; \lambda+k)| \geq \frac{c}{2}(1+|k|)^{m} \quad \text { for every } k \in\left(\mathbb{N}^{p}\right)^{*},
$$

where $c$ is the positive constant in (2.4).

Proof. Since

$$
\begin{aligned}
\widetilde{Q}(y ; \lambda+k)= & \widetilde{Q}(0 ; \stackrel{\circ}{\lambda}+k)+\int_{0}^{1} D_{t}\{\widetilde{Q}(t y ; \stackrel{\circ}{\lambda}+k+t(\lambda-\stackrel{\circ}{\lambda}))\} d t \\
= & \widetilde{Q}(0 ; \stackrel{\circ}{\lambda}+k)+\int_{0}^{1}\left\{\left(D_{y} \widetilde{Q}\right)(t y ; \stackrel{\circ}{\lambda}+k+t(\lambda-\stackrel{\circ}{\lambda})) \cdot y\right. \\
& \left.\quad+\left(D_{\lambda} \widetilde{Q}\right)(t y ; \dot{\circ}+k+t(\lambda-\stackrel{\circ}{\lambda})) \cdot(\lambda-\stackrel{\circ}{\lambda})\right\} d t,
\end{aligned}
$$

assuming that $y$ and $\lambda$ are in a bounded neighborhood of 0 and $\stackrel{i}{\lambda}$ respectively, we have

$$
\begin{array}{r}
|\widetilde{Q}(y ; \lambda+k)| \geq|\widetilde{Q}(0 ; \dot{\lambda}+k)|-\int_{0}^{1}\left\{\left|\left(D_{y} \widetilde{Q}\right)(t y ; \stackrel{\circ}{\lambda}+k+t(\lambda-\stackrel{\circ}{\lambda}))\right||y|\right. \\
\left.+\left|\left(D_{\lambda} \widetilde{Q}\right)(y ; \stackrel{\circ}{\lambda}+k+t(\lambda-\stackrel{\circ}{\lambda}))\right||\lambda-\stackrel{\circ}{\lambda}|\right\} d t \\
\geq c(1+|k|)^{m}-M(1+|k|)^{m}|y|-M(1+|k|)^{m-1}|\lambda-\stackrel{\circ}{\lambda}|
\end{array}
$$

for some constant $M$. Thus, if we take $\epsilon$ as $M \epsilon \leq c / 4$, then we get the desired estimate. 
Lemma 4.2. There exist a simple closed curve $\Gamma_{i}$ in $\mathbb{C}$ enclosing $\stackrel{\circ}{\lambda}_{i}$ for each $i=1, \ldots, p$, a domain $\Omega^{\prime}$ including $y=0$ in $\mathbb{C}^{q}$, and a constant $c_{0}>0$ such that the following holds.

i) If $y \in \overline{\Omega^{\prime}}$, then $\lambda_{0, i}(y)$ lies inside $\Gamma_{i}$, for every $i=1, \ldots, p$.

ii) For every $\zeta \in \Gamma:=\Gamma_{1} \times \cdots \times \Gamma_{p}$, every $y \in \Omega^{\prime}$, and every $k \in \mathbb{N}^{p}$,

$$
|\widetilde{Q}(y ; \zeta+k)| \geq c_{0}(1+|k|)^{m}
$$

Note that $k=0$ is allowed in (4.6) and is not allowed in (4.5), while $\zeta=\stackrel{\AA}{\text { is }}$ not allowed in (4.6) and $\lambda=\stackrel{\circ}{\lambda}$ is allowed in (4.5),

Proof. By the assumption $\left(\mathrm{A}_{0}\right)$ with $\mu=0$, the Fuchsian characteristic polynomial $\widetilde{Q}(y ; \lambda)$ has terms $a_{i}(y) \lambda_{i}^{m}$ with $a_{i}(0) \neq 0$, for every $i$. Especially, $g\left(\lambda_{1}\right):=$ $\widetilde{Q}\left(0 ; \lambda_{1}, \stackrel{\circ}{\lambda}_{2}, \ldots, \stackrel{\circ}{\lambda}_{p}\right)$ is a nonzero polynomial of $\lambda_{1}$, which has a zero $\stackrel{\circ}{\lambda}_{1}$. Hence, we can take $r \in(0, \epsilon]$ such that $g\left(\lambda_{1}\right) \neq 0$ for $0<\left|\lambda_{1}-\grave{\lambda}_{1}\right|<r$. We take a simple closed curve $\Gamma_{1}$ inside this region. Hence, we have

$$
\widetilde{Q}\left(0 ; \zeta_{1}, \stackrel{\circ}{\lambda}_{2}, \ldots, \stackrel{\circ}{\lambda}_{p}\right) \neq 0 \quad \text { for } \zeta_{1} \in \Gamma_{1}
$$

Then, there exists $r^{\prime} \in(0, \epsilon]$ such that $\widetilde{Q}\left(0 ; \zeta_{1}, \lambda_{2}, \ldots, \lambda_{p}\right) \neq 0$ for $\zeta_{1} \in \Gamma_{1}$ and $\lambda_{i} \in B_{r^{\prime}}\left(\grave{\lambda}_{i}\right) \subset \mathbb{C}(i \geq 2)$, where $\epsilon$ is the positive constant in Lemma 4.1. We take each simple closed curve $\Gamma_{i}$ enclosing $\stackrel{\circ}{\lambda}_{i}$ inside $B_{r^{\prime}}\left(\stackrel{\curlywedge}{\lambda}_{i}\right)$ for $i \geq 2$. Hence, we have

$$
\widetilde{Q}(0 ; \zeta) \neq 0 \quad \text { for } \zeta \in \Gamma \text {. }
$$

Finally, we can take a domain $\Omega^{\prime} \subset B_{\epsilon}(0)$ including $y=0$ such that

i) if $y \in \overline{\Omega^{\prime}}$, then $\lambda_{0, i}(y)$ lies inside $\Gamma_{i}$ for every $i=1, \ldots, p$,

ii) $\widetilde{Q}(y ; \zeta) \neq 0$ if $\zeta \in \Gamma$ and $y \in \overline{\Omega^{\prime}}$.

Since $\overline{\Omega^{\prime}} \times \Gamma$ is compact, the inequality (4.6) holds for $k=0$ by taking a sufficiently small $c_{0}>0$. For $k \neq 0$, the inequality holds by Lemma 4.1. 
By the proof, we may assume that the diameters of $\Gamma_{i}$ are smaller than $1 / p$, which is not really necessary, but convenient for later argument.

Now, we can solve (4.4).

Proposition 4.1. There exist a domain $\omega$ including $x=0$ in $\mathbb{C}^{p}$, a domain $\Omega \subset \Omega^{\prime}$ including $y=0$ in $\mathbb{C}^{q}$, and a unique $V \in \mathscr{O}(\omega \times \Omega \times \Gamma)$ such that (4.4) holds on $\omega \times \Omega \times \Gamma$.

Proof. First note that $\widetilde{P}:=p\left(x, y ; x D_{x}+\zeta, D_{y}\right)$ is a Fuchsian operator in the variables $(x, y, \zeta)$ with weight 0 with respect to $x$. Its Fuchsian characteristic polynomial is $\widetilde{Q}(y ; \lambda+\zeta)$. Also note that the right-hand side of (4.4) is holomorphic in a neighborhood of $y=0$ and $\zeta \in \Gamma$.

Fix $\zeta_{*} \in \Gamma$ arbitrary. Then, considering $\left(0,0 ; \zeta_{*}\right)$ as the origin, we can apply Theorem 3.1 to $\widetilde{P}$ by (4.6). Hence, there exists a unique holomorphic $V$ in a neighborhood of $\left(0,0 ; \zeta_{*}\right)$. Since $V$ is unique and $\zeta_{*} \in \Gamma$ is arbitrary, we have the desired $V$.

Since

$$
\begin{aligned}
\int_{\Gamma} \mathcal{G}(\zeta ; x) \frac{\widetilde{Q}(y ; \zeta)}{\left(\zeta_{1}-\lambda_{0,1}(y)\right) \cdots\left(\zeta_{p}-\lambda_{0, p}(y)\right)} d \zeta \\
=(2 \pi \sqrt{-1})^{p} \mathcal{G}\left(\lambda_{0}(y) ; x\right) \widetilde{Q}\left(y ; \lambda_{0}(y)\right) \equiv 0,
\end{aligned}
$$

the distribution $u \in \mathscr{D}_{+}^{\prime}(\mathcal{V} ; \mathscr{O}(\Omega)) \cap C^{\infty}\left(\mathcal{V}_{+} ; \mathscr{O}(\Omega)\right)$ defined by (4.1) is a solution of $\mathcal{P} u=0$, where $\mathcal{V}:=\omega \cap \mathbb{R}^{p}$.

\section{Support of the solution}

In this section, we show that $(0,0) \in \operatorname{supp} u$ for the solution constructed in the previous section, which completes the proof of our theorem. 
For every $N \in \mathbb{N}$, we can expand $V(x, 0 ; \zeta)$ as

$$
\begin{aligned}
& V(x, 0 ; \zeta)=\sum_{\gamma \in \mathbb{N}^{p} ;|\gamma| \leq N} V_{\gamma}(\zeta) x^{\gamma}+\widetilde{V}_{N+1}(x ; \zeta), \\
& V_{\gamma} \in \mathscr{O}(\Gamma),|\gamma| \leq N ; \quad \widetilde{V}_{N+1} \in \mathscr{O}(\omega \times \Gamma), \\
& \sup _{\zeta \in \Gamma}\left|\widetilde{V}_{N+1}(x ; \zeta)\right|=O\left(|x|^{N+1}\right) \quad \text { as }|x| \rightarrow 0 .
\end{aligned}
$$

Corresponding to this expansion, we have

$$
\begin{aligned}
u(x, 0) & =\sum_{\gamma \in \mathbb{N}^{p} ;|\gamma| \leq N} u_{\gamma}(x) x^{\gamma}+\widetilde{u}_{N+1}(x), \\
u_{\gamma}(x) & =\int_{\Gamma} \mathcal{G}(\zeta ; x) V_{\gamma}(\zeta) d \zeta \in \mathscr{S}_{+}^{\prime}\left(\mathbb{R}^{p}\right),|\gamma| \leq N \\
\widetilde{u}_{N+1}(x) & =\int_{\Gamma} \mathcal{G}(\zeta ; x) \widetilde{V}_{N+1}(x ; \zeta) d \zeta \in \mathscr{D}_{+}^{\prime}(\mathcal{V}) .
\end{aligned}
$$

By noting $p\left(0, y ; \zeta, D_{y}\right)=\widetilde{Q}(y ; \zeta)$, we can see from (4.4) that

$$
V(0, y ; \zeta)=\frac{1}{\left(\zeta_{1}-\lambda_{0,1}(y)\right) \cdots\left(\zeta_{p}-\lambda_{0, p}(y)\right)},
$$

and hence we have

$$
V_{0}(\zeta)=V(0,0 ; \zeta)=\frac{1}{\left(\zeta_{1}-\stackrel{\circ}{\lambda}_{1}\right) \ldots\left(\zeta_{p}-\stackrel{\circ}{\lambda}_{p}\right)},
$$

which implies

$$
u_{0}(x)=(2 \pi \sqrt{-1})^{p} \mathcal{G}(\stackrel{\circ}{\lambda} ; x) .
$$

Clearly $0 \in \operatorname{supp} u_{0}$. In order to show that $0 \in \operatorname{supp} u(x, 0)$, we observe the order of $\langle\chi(x) u(x, 0), \exp (-\langle x\rangle / \epsilon)\rangle_{x}$ as $\epsilon \rightarrow+0$, where $\chi \in C_{0}^{\infty}(\mathcal{V})$ and $\langle x\rangle:=$ $\sum_{i=1}^{p} x_{i}$.

Lemma 5.1. Let $M \geq 0$, and $w \in C_{+}^{0}\left(\mathbb{R}^{p}\right)$. If there exist constants $C$ and $K$ such that $|x|^{-M}|w(x)| \leq C(1+|x|)^{K}$ on $\mathbb{R}_{+}^{p}$, then we have $\langle w(x), \exp (-\langle x\rangle / \epsilon)\rangle_{x}=$ $O\left(\epsilon^{M+p}\right)$ as $\epsilon \rightarrow+0$.

Proof. We have

$$
\begin{aligned}
\left|\langle w(x), \exp (-\langle x\rangle / \epsilon)\rangle_{x}\right| & \leq C \int_{\mathbb{R}_{+}^{p}}|x|^{M}(1+|x|)^{K} \exp (-\langle x\rangle / \epsilon) d x \\
& \leq C \int_{\mathbb{R}_{+}^{p}}|y|^{M}(1+\epsilon|y|)^{K} \exp (-\langle y\rangle) d y \epsilon^{M+p} \\
& =O\left(\epsilon^{M+p}\right) .
\end{aligned}
$$


Lemma 5.2. If $w \in \mathscr{S}_{+}^{\prime}\left(\mathbb{R}^{p}\right)$ and $w=0$ in a neighborhood of $x=0$, then we have

$$
\langle w(x), \exp (-\langle x\rangle / \epsilon)\rangle_{x}=O\left(\epsilon^{\infty}\right) \quad \text { as } \epsilon \rightarrow+0
$$

In order to prove this lemma, we give a supplementary lemma.

Lemma 5.3. (1) Let $a \in[0, \infty)$ and set $f_{a, \epsilon}(t):=|t|^{a} \exp (-t / \epsilon)$. If $\delta_{1}, \delta_{2}>0$, then there exists a constant $\epsilon_{0}=\epsilon_{0}\left(a, \delta_{1}, \delta_{2}\right)>0$ such that for $0<\epsilon \leq \epsilon_{0}$ we have

$$
\begin{gathered}
\sup _{t \geq \delta_{1}} f_{a, \epsilon}(t)=f_{a, \epsilon}\left(\delta_{1}\right)=\delta_{1}^{a} \exp \left(-\delta_{1} / \epsilon\right), \\
\sup _{t \geq-\delta_{2}} f_{a, \epsilon}(t)=f_{a, \epsilon}\left(-\delta_{2}\right)=\delta_{2}^{a} \exp \left(\delta_{2} / \epsilon\right) .
\end{gathered}
$$

(2) Let $\alpha \in \mathbb{N}^{p}$ and set $g_{\alpha, \epsilon}(x):=\left|x^{\alpha}\right| \exp (-\langle x\rangle / \epsilon)$. Let $r>0$ and set

$$
A_{r}:=\left\{x \in \mathbb{R}^{p}|| x \mid>p^{2} r, x_{i}>-r(i=1, \ldots, p)\right\} .
$$

Then, there exist constants $\epsilon_{0}=\epsilon_{0}(\alpha, r)>0$ and $C_{0}=C_{0}(\alpha, r)>0$ such that

$$
\sup _{x \in \overline{A_{r}}} g_{\alpha, \epsilon}(x) \leq C_{0} \exp (-r / \epsilon) \quad \text { for } 0<\epsilon \leq \epsilon_{0} .
$$

Proof. (1) is elementary and the proof is omitted.

(2) First, we show that if $x \in \overline{A_{r}}$, then there exists $i_{0}$ such that $x_{i_{0}} \geq p r$. For each $x \in \overline{A_{r}}$, take $i_{0}$ as $\max _{1 \leq i \leq p}\left|x_{i}\right|=\left|x_{i_{0}}\right|$. Then, $\left|x_{i_{0}}\right| \geq|x| / \sqrt{p} \geq p r$. Since, $x_{i_{0}} \geq-r$, we have $x_{i_{0}} \geq p r$.

Hence, using (1), we have

$$
\begin{aligned}
\sup _{x \in \overline{A_{r}}} g_{\alpha, \epsilon}(x) & \leq \max _{1 \leq i_{0} \leq p}\left\{\sup _{x_{i_{0}} \geq p r ; x_{i} \geq-r\left(i \neq i_{0}\right)} g_{\alpha, \epsilon}(x)\right\} \\
& \leq \max _{1 \leq i_{0} \leq p}\left\{\sup _{x_{i_{0}} \geq p r} f_{\alpha_{i_{0}}, \epsilon}\left(x_{i_{0}}\right) \prod_{i \neq i_{0}} \sup _{x_{i} \geq-r} f_{\alpha_{i}, \epsilon}\left(x_{i}\right)\right\} \\
& \leq \max _{1 \leq i_{0} \leq p}\left\{(p r)^{\alpha_{i_{0}}} \exp (-p r / \epsilon) \prod_{i \neq i_{0}} r^{\alpha_{i}} \exp (r / \epsilon)\right\} \\
& \leq C_{0} \exp (-r / \epsilon) .
\end{aligned}
$$


Proof of Lemma 5.2. Take $r>0$ such that $w(x)=0$ in a neighborhood of $\overline{B_{p^{2} r}(0)}$. Then, supp $w \subset A_{r}$. Take $\chi \in C^{\infty}\left(\mathbb{R}^{p}\right)$ such that $\chi(x)=1$ in a neighborhood of $\operatorname{supp} w$ and $\operatorname{supp} \chi \subset \overline{A_{r}}$. Then,

$$
\langle w(x), \exp (-\langle x\rangle / \epsilon)\rangle_{x}=\langle w(x), \chi(x) \exp (-\langle x\rangle / \epsilon)\rangle_{x}
$$

We have only to show that $\phi_{\epsilon}(x):=\chi(x) \exp (-\langle x\rangle / \epsilon)$ satisfies $\phi_{\epsilon}=O\left(\epsilon^{\infty}\right)$ in $\mathscr{S}\left(\mathbb{R}^{p}\right)$.

For every $N \in \mathbb{N}$ and $\beta \in \mathbb{N}^{p}$, there exist constants $C, C^{\prime}>0$ independent of $\epsilon>0$ such that

$$
\begin{aligned}
\sup _{x \in \mathbb{R}^{p}}(1+|x|)^{N}\left|D_{x}^{\beta} \phi_{\epsilon}(x)\right| & \leq C \sum_{|\alpha| \leq N ; 0 \leq j \leq|\beta|} \epsilon^{-j} \sup _{x \in \overline{A_{r}}}\left|x^{\alpha}\right| \exp (-\langle x\rangle / \epsilon) \\
& \leq C^{\prime} \epsilon^{-|\beta|} \exp (-r / \epsilon) \quad \text { for } 0<\epsilon \leq \epsilon_{0}
\end{aligned}
$$

by Lemma $5.3(2)$.

We set $r_{i}:=\min \left\{\operatorname{Re} \zeta_{i} \mid \zeta_{i} \in \Gamma_{i}\right\}$ and take $\delta_{i} \in \mathbb{N}$ such that $\delta_{i} \geq \max \left\{-r_{i}, 0\right\}$. Note that $\operatorname{Re} \zeta_{i}+\delta_{i} \geq 0$ for $\zeta_{i} \in \Gamma_{i}$. Assume that $N+1 \geq|\delta|$.

Lemma 5.4. For $\chi \in C_{0}^{\infty}(\mathcal{V})$, we have

$$
\left\langle\chi(x) \widetilde{u}_{N+1}(x), \exp (-\langle x\rangle / \epsilon)\right\rangle_{x}=O\left(\epsilon^{N+1-|\delta|+p}\right) \quad \text { as } \epsilon \rightarrow+0 .
$$

Proof. $\quad$ Since $D_{x}^{\delta} \mathcal{G}(\zeta+\delta ; x)=\mathcal{G}(\zeta ; x)$, we have

$$
\begin{aligned}
\langle\chi(x) & \left.\widetilde{u}_{N+1}(x), \exp (-\langle x\rangle / \epsilon)\right\rangle_{x} \\
= & \int_{\Gamma}\left\langle\mathcal{G}(\zeta ; x) \widetilde{V}_{N+1}(x ; \zeta), \chi(x) \exp (-\langle x\rangle / \epsilon)\right\rangle_{x} d \zeta \\
= & \int_{\Gamma}\left\langle D_{x}^{\delta} \mathcal{G}(\zeta+\delta ; x), \widetilde{V}_{N+1}(x ; \zeta) \chi(x) \exp (-\langle x\rangle / \epsilon)\right\rangle_{x} d \zeta \\
= & \int_{\Gamma}\left\langle\mathcal{G}(\zeta+\delta ; x),\left(-D_{x}\right)^{\delta}\left\{\widetilde{V}_{N+1}(x ; \zeta) \chi(x) \exp (-\langle x\rangle / \epsilon)\right\}\right\rangle_{x} d \zeta \\
= & \int_{\Gamma}\left\langle\mathcal{G}(\zeta+\delta ; x), \sum_{j=0}^{|\delta|} W_{j}(x ; \zeta) \epsilon^{-j} \exp (-\langle x\rangle / \epsilon)\right\rangle_{x} d \zeta,
\end{aligned}
$$

where $W_{j}(x ; \zeta):=\sum_{|\gamma|=|\delta|-j}\left(\begin{array}{l}\delta \\ \gamma\end{array}\right)\left(-D_{x}\right)^{\gamma}\left\{\widetilde{V}_{N+1}(x ; \zeta) \chi(x)\right\} \in C_{0}^{\infty}(\mathcal{V} ; \mathscr{O}(\Gamma))$ and $\sup _{\zeta \in \Gamma}\left|W_{j}(x ; \zeta)\right|=O\left(|x|^{N+1-|\delta|+j}\right)$. Since $\operatorname{Re} \zeta_{i}+\delta_{i} \geq 0$ if $\zeta_{i} \in \Gamma_{i}$, we have

$$
w_{j}(x):=\int_{\Gamma} \mathcal{G}(\zeta+\delta ; x) W_{j}(x ; \zeta) d \zeta \in C_{0}^{0}(\mathcal{V})
$$


and $w_{j}(x)=O\left(|x|^{N+1-|\delta|+j}\right)$.

By Lemma 5.1, we have

$$
\begin{aligned}
\left\langle\chi(x) \widetilde{u}_{N+1}(x), \exp (-\langle x\rangle / \epsilon)\right\rangle_{x} & =\sum_{j=0}^{|\delta|}\left\langle w_{j}(x), \exp (-\langle x\rangle / \epsilon)\right\rangle_{x} \epsilon^{-j} \\
& =O\left(\epsilon^{N+1-|\delta|+p}\right) .
\end{aligned}
$$

Since $\mathcal{G}(\zeta ; x) x^{\gamma}=\mathcal{C}_{\gamma}(\zeta+\gamma) \mathcal{G}(\zeta+\gamma ; x)$ by Lemma $3.2(1)$, we have

$$
\begin{aligned}
\left\langle u_{\gamma}(x) x^{\gamma}, \exp (-\langle x\rangle / \epsilon)\right\rangle_{x} & =\int_{\Gamma} \mathcal{C}_{\gamma}(\zeta+\gamma) \epsilon^{\langle\zeta+\gamma\rangle+p} V_{\gamma}(\zeta) d \zeta \\
& =O\left(\epsilon^{\langle r\rangle+|\gamma|+p}\right)
\end{aligned}
$$

using Lemma 3.2(3). Especially,

$$
\left\langle u_{0}(x), \exp (-\langle x\rangle / \epsilon)\right\rangle_{x}=(2 \pi \sqrt{-1})^{p} \epsilon^{\langle\grave{\lambda}\rangle+p} .
$$

Take $\chi_{0} \in C_{0}^{\infty}(\mathcal{V})$ satisfying $\chi_{0}(x)=1$ in a neighborhood of $x=0$. Then,

$$
\left\langle\chi_{0}(x) \widetilde{u}_{N+1}(x), \exp (-\langle x\rangle / \epsilon)\right\rangle_{x}=O\left(\epsilon^{N+1-|\delta|+p}\right)
$$

by Lemma 5.4. By Lemma 5.2, we have

$$
\left\langle\left(\chi_{0}(x)-1\right) \sum_{|\gamma| \leq N} u_{\gamma}(x) x^{\gamma}, \exp (-\langle x\rangle / \epsilon)\right\rangle_{x}=O\left(\epsilon^{\infty}\right) .
$$

Since we may assume that the diameter of $\Gamma_{i}$ is smaller than $1 / p$, we have $r_{i}>$ $\operatorname{Re} \stackrel{\circ}{\lambda}_{i}-1 / p$, that is $\langle r\rangle+1>\operatorname{Re}\langle\grave{\lambda}\rangle$. Hence, if we take $N \in \mathbb{N}$ such that $N+1>$

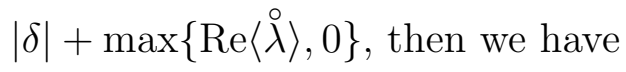

$$
\begin{aligned}
&\left\langle\chi_{0}(x) u(x, 0), \exp (-\langle x\rangle / \epsilon)\right\rangle_{x} \\
&=\left\langle\chi_{0}(x) \sum_{|\gamma| \leq N} u_{\gamma}(x) x^{\gamma}, \exp (-\langle x\rangle / \epsilon)\right\rangle_{x} \\
& \quad+\left\langle\chi_{0}(x) \widetilde{u}_{N+1}(x), \exp (-\langle x\rangle / \epsilon)\right\rangle_{x} \\
&=\sum_{|\gamma| \leq N}\left\langle u_{\gamma}(x) x^{\gamma}, \exp (-\langle x\rangle / \epsilon)\right\rangle_{x} \\
& \quad+\left\langle\left(\chi_{0}(x)-1\right) \sum_{|\gamma| \leq N} u_{\gamma}(x) x^{\gamma}, \exp (-\langle x\rangle / \epsilon)\right\rangle_{x} \\
& \quad+\left\langle\chi_{0}(x) \widetilde{u}_{N+1}(x), \exp (-\langle x\rangle / \epsilon)\right\rangle_{x} \\
&=(2 \pi \sqrt{-1})^{p} \epsilon^{\langle\lambda\rangle+p}+o\left(\epsilon^{\langle i \lambda\rangle+p}\right) .
\end{aligned}
$$


On the other hand, if $0 \notin \operatorname{supp} u(\cdot, 0)$, then

$$
\langle\chi(x) u(x, 0), \exp (-\langle x\rangle / \epsilon)\rangle_{x}=O\left(\epsilon^{\infty}\right)
$$

by Lemma 5.2. Thus, we have $0 \in \operatorname{supp} u(\cdot, 0)$, and hence $(0,0) \in \operatorname{supp} u$.

\section{$6 \quad$ Final remarks}

In the case of one Fuchsian variable, there exist no sufficiently smooth nullsolutions. In the case of several Fuchsian variables, however, some simple examples show that there may exist $C^{\infty}$ null-solutions, and there may not exist sufficiently smooth null-solutions.

Example 6.1. Let $m=1$. Also let $q=0$ for the sake of simplicity. Consider $\mathcal{P}=\sum_{j=1}^{p} a_{j} x_{j} D_{x_{j}}$, where $a_{j}$ are real constants. If $\lambda \in \mathbb{C}^{p}$ satisfies $\sum_{j=1}^{p} a_{j} \lambda_{j}=0$, then $u(x)=\mathcal{G}(\lambda ; x)$ is a distribution null-solution.

(1) If there exist $i$ and $j$ such that $a_{i} a_{j}<0$, then for every $M \in \mathbb{R}$, we can take $\lambda \in \mathbb{R}^{p}$ such that $\sum_{j=1}^{p} a_{j} \lambda_{j}=0$ and $\min \lambda_{j}>M$. Hence, we have null-solutions as smooth as we desire. Moreover, $\mathcal{P}$ has a $C^{\infty}$ null-solution. In fact, there exist $\rho_{j}>0 \quad(j=1, \ldots, p)$ such that $\sum_{j=1}^{p} \rho_{j} a_{j}=0$, and hence if $\phi \in C_{+}^{\infty}(\mathbb{R})$ and $0 \in \operatorname{supp} \phi \subset \overline{\mathbb{R}_{+}}$, then

$$
u(x):= \begin{cases}\phi\left(x_{1}^{\rho_{1}} \ldots x_{p}^{\rho_{p}}\right) & \left(x \in \mathbb{R}_{+}^{p}\right) \\ 0 & \text { (otherwise) }\end{cases}
$$

is a $C^{\infty}$ null-solution for $\mathcal{P}$ at $x=0$ with respect to $\overline{\mathbb{R}_{+}^{p}}$.

This example illustrates also that the assumption $\left(A_{0}\right)$ is not necessary for the existence of distribution null-solutions, since it is easy to show that $\mathcal{P}$ does not satisfy the condition $\left(\mathrm{A}_{0}\right)$.

(2) If $a_{j}>0$ for every $j$, then $\mathcal{P}$ has no $C^{0}$ null-solution. In fact, let $\epsilon>0$ and $u \in$ $C_{+}^{0}\left(B_{\epsilon}(0)\right)$ satisfy $\mathcal{P} u=0$ on $B_{\epsilon}(0)$. For every $b=\left(b_{1}, \ldots, b_{p}\right) \in \mathbb{R}_{+}^{p}$, we set $\phi_{b}(t):=$ $u\left(b_{1} t^{a_{1}}, \ldots, b_{p} t^{a_{p}}\right)$. We have $D_{t} \phi_{b}(t)=\sum_{j=1}^{p}\left(D_{x_{j}} u\right)\left(b_{1} t^{a_{1}}, \ldots, b_{p} t^{a_{p}}\right) b_{j} a_{j} t^{a_{j}-1}=$ $(1 / t)(\mathcal{P} u)\left(b_{1} t^{a_{1}}, \ldots, b_{p} t^{a_{p}}\right)=0$ on $t \in\left(0, \epsilon_{b}\right)$ in distribution sense, where

$$
\epsilon_{b}:=\max \left\{t>0 \mid\left(b_{1} t^{a_{1}}, \ldots, b_{p} t^{a_{p}}\right) \in B_{\epsilon}(0)\right\} .
$$


Hence, $\phi_{b}(t)$ is a constant on $\left(0, \epsilon_{b}\right)$. Since $\phi_{b}(t) \rightarrow 0$ as $t \rightarrow+0$, we have $u\left(b_{1} t^{a_{1}}, \ldots, b_{p} t^{a_{p}}\right)=0$ for $t \in\left(0, \epsilon_{b}\right)$. Since $b \in \mathbb{R}_{+}^{p}$ is arbitrary and $u$ is continuous, we have $u(x)=0$ on $B_{\epsilon}(0)$. Note that $\mathcal{P}$ satisfies the condition $\left(\mathrm{A}_{0}\right)$ in this case.

As a matter of fact, if $p=2$, this operator is one of the simplest examples of the operators considered by N. S. Madi in [9], which have no sufficiently smooth null-solutions by Proposition 2 in [9].

Acknowledgements The authors are very grateful for Professor Hidetoshi Tahara (Sophia University, Japan) for his valuable advice.

\section{References}

[1] M. S. Baouendi and C. Goulaouic, Cauchy problem with characteristic initial hypersurface, Comm. Pure Appl. Math., 26(1973), 455-475.

[2] A. Cauchy, Mémoire sur un théorème fondamental dans le calcul intégral, $C$. R. Acad. Sci. Paris, 39(1842), 1020-1026. (see Extrait No167 des Oeuvres Complétes de A. Cauchy T. VI)

[3] A. Cauchy, Mémoire sur l'emploi du calcul des limites dans l'intégration des équations aux dérivées partielles, C. R. Acad. Sci. Paris, 40(1842), 44-59. (voire Extrait $\mathrm{N}^{\circ} 170$ des Oeuvres Compléte de A. Cauchy T.VII).

[4] A. Cauchy, Mémoire sur l'application du calcul des limites à l'intégration d'un système d'équations aux dérivées partielles, C. R. Acad. Sci. Paris, 40(1842), 85-101. (voire Extrait No171 des Oeuvres Compléte de A. Cauchy T.VII).

[5] K. Igari, Non unicité dans le problème de Cauchy caractéristique — cas de type de Fuchs, J. Math. Kyoto Univ., 25(1985), 341-355.

[6] K. Igari, Remarks on null solutions of linear partial differential equations, $J$. Math. Kyoto Univ., 27(1987), 47-59. 
[7] H. Komatsu, Irregularity of characteristic elements and construction of null solutions, J. Fac. Sci. Univ. Tokyo, sect. IA Math., 23(1976), 297-342.

[8] N. S. Madi, Problème de Goursat holomorphe à variables fuchsiennes, Bull. Sc. Math., 2e série, 111(1987), 291-312.

[9] N. S. Madi, Problème de Cauchy a deux variables Fuchsiennes, Can. J. Math., 43(1991), 1036-1044.

[10] N. S. Madi, Solutions locales pour des opérateurs holomorphes fuchsiens en plusieurs variables, Ann. Math. Pura Appl. (IV), 163(1993), 1-15.

[11] T. Mandai, Existence of $C^{\infty}$ null-solutions and behavior of bicharacteristic operators with $C^{\infty}$ coefficients, Japan. Math. (N.S), 15(1989), 53-63.

[12] T. Mandai, Existence of distribution null-solutions for every Fuchsian partial differential operator, J. Math. Sci. Univ. Tokyo, 5(1998), 1-18.

[13] T. Mandai, The method of Frobenius to Fuchsian partial differential equations, J. Math. Soc. Japan, 52(2000), 645-672.

[14] S. Mizohata, Solutions nulles et solutions non analytiques, J. Math. Kyoto Univ., 1(1962), 271-302.

[15] I. Petrowsky, Sur l'analyticité des solutions des systèmes d'équations différentielles, Mat. Sbornik, 5(1939), 3-70.

[16] H. Tahara, Cauchy problems for Fuchsian hyperbolic partial differential equations, Proc. Japan Acad. Ser. A Math. Sci., 54(1978), 92-96.

[17] H. Tahara, Fuchsian type equations and Fuchsian hyperbolic equations, Japan. J. Math. (N.S.), 5(1979), 245-347.

[18] H. Tahara, Singular hyperbolic systems, V. Asymptotic expansions for Fuchsian hyperbolic partial differential equation, J. Math. Soc. Japan, 36(1984), 449-473. 
[19] H. Tahara, Singular hyperbolic systems, VI. Asymptotic analysis for Fuchsian hyperbolic hyperbolic equations in Gevrey classes, J. Math. Soc. Japan, 39(1987), 551-580.

[20] H. Tahara, Singular hyperbolic systems, VII. Asymptotic analysis for Fuchsian hyperbolic equations in Gevrey classes 2, J. Math. Soc. Japan, 15(1989), 275307.

[21] H. Tahara, Singular hyperbolic systems, VIII. On the Well-posedness in Gevrey classes for Fuchsian hyperbolic equations, J. Fac. Sci., Univ. Tokyo, Sec. IA, 39(1992), 555-582.

Laboratoire de Mathématiques,

UNiversity of DJILALI Liabès,

B.P. 89, 22000 Sidi Bel AbBÈs, Algeria

E-mail address: mkbelarbi@yahoo.com

Research Center for Physics and Mathematics,

Osaka Electro-Communication University,

18-8 Hatsu-Cho, Neyagawa-Shi,

OSAKA 572-8530, JAPAN

E-mail address: mandai@isc.osakac.ac.jp

Laboratoire de Mathématiques,

UNIVERSity of DJILALI LiabÈs,

B.P. 89, 22000 Sidi Bel AbBÈs, Algeria

E-mail address: mechab@univ-sba.dz 